\title{
The Drosophila short gastrulation gene prevents Dpp from autoactivating and suppressing neurogenesis in the neuroectoderm
}

\author{
Brian Biehs, Vincent François, ${ }^{1}$ and Ethan Bier ${ }^{2}$ \\ Department of Biology and Center for Molecular Genetics, University of California, San Diego, \\ La Jolla, California 92093 USA
}

\begin{abstract}
The short gastrulation (sog) gene is expressed in broad lateral stripes comprising the neuroectoderm of the Drosophila blastoderm embryo. sog encodes a predicted secreted protein that functions nonautonomously to antagonize the activity of the TGF- $\beta$-like Decapentaplegic (Dpp) signaling pathway in the dorsal region of the embryo. Recently, it has been shown that $\operatorname{sog}$ and $d p p$ are functionally equivalent to their respective Xenopus homologs chordin and BMP-4. In this report we provide the first direct evidence that sog plays a local role in the lateral region of the blastoderm embryo to oppose Dpp activity in the neuroectoderm. In the dorsal region, Dpp signaling both suppresses neurogenesis and maintains expression of genes that promote dorsal cell fates (dorsalization). We show that Dpp also can perform both of these functions in the neuroectoderm. In wild-type embryos, the ability of Dpp to induce expression of dorsal markers including itself (autoactivation) in the neuroectoderm is blocked by sog. We propose that Sog protects the neuroectoderm from an invasive positive feedback loop created by Dpp diffusion and autoactivation. We show that the two functions of Dpp signaling, neural suppression and dorsalization, are triggered by distinct thresholds of Dpp activity. Epistasis experiments reveal that all observed sog activity can be accounted for by Sog functioning as a dedicated Dpp antagonist. Finally, we provide evidence that Sog functions as a diffusible morphogen in the blastoderm embryo. These data strongly support the view that the primary phylogenetically conserved function of the Drosophila sog and dpp genes and the homologous Xenopus chordin and BMP-4 genes is to subdivide the primitive embryonic ectoderm into neural versus non-neural domains.
\end{abstract}

[Key Words: short gastrulation (sog); decapentaplegic $(d p p)$; TGF- $\beta$; morphogen; neuroectoderm; dorsal-ventral axis; evolution; Drosophila]

Received August 13, 1996; revised version accepted September 25, 1996.

The vertebrate bone morphogenetic proteins (BMPs) define a subgroup of the transforming growth factor- $\beta$ (TGF- $\beta$ ) superfamily capable of organizing undifferentiated mesenchymal cells into bone tissue (Rosen and Thies 1992; Kingsley 1994). At least two mouse BMPs are also individually important during development as mutations in these genes (short ear and brachypodism) lead to defects in bone formation and induction between germ layers (Kingsley et al. 1992; Storm et al. 1994). Furthermore, as described below, BMP-4 and its Drosophila homolog Dpp play analogous roles during dorsal-ventral (D-V) axis formation and neurogenesis in Xenopus and Drosophila (Graff et al. 1994; Maeno et al. 1994; Holley et al. 1995; Schmidt et al. 1995a).

Establishment of the D-V axis in Drosophila is deter-

${ }^{1}$ Present address: CNRS, 340033 Montpellier Cedex1, France. ${ }^{2}$ Corresponding author. mined by a cascade of maternally acting genes functioning in both the oocyte and surrounding follicle cells. U1timately these genes establish a nuclear gradient of the NF- $\mathrm{kB}$-related transcription factor Dorsal (Roth et al. 1989; Rushlow et al. 1989; Steward 1989). The Dorsal nuclear gradient is directly responsible for subdividing the embryo into three primary territories of zygotic gene expression: (1) a ventral zone comprising the presumptive mesoderm, (2) a lateral zone comprising the neuroectoderm, and (3) a dorsal zone giving rise to dorsal ectoderm and amnioserosa. Although Dorsal activates expression of genes in ventral and lateral regions, it specifies the dorsal zone by repressing ventral and lateral expression of key zygotic dorsal group (ZDG) genes, including decapentaplegic (dpp), zerknüllt (zen), tolloid $(t l d)$, and twisted gastrulation $(t s g)$, which are required for the differentiation of dorsal tissue types.

The $d p p$ gene, which encodes a protein (Padgett et al. 1987) most similar to vertebrate BMP-2 and BMP-4, 
plays a key role in patterning the dorsal region. Embryos lacking $d p p$ function, which exhibit the most severely ventralized phenotype observed among ZDG mutants (Ray et al. 1991; Arora and Nüsslein-Volhard 1992), fail to make any dorsally derived structures (Irish and Gelbart 1987). Consistent with its developmental function, $d p p$ is expressed throughout the dorsal region of the early precellular blastoderm embryo (St. Johnson and Gelbart 1987). A variety of evidence suggests that Dpp functions as a morphogen with different levels of Dpp specifying distinct developmental fates in dorsal and lateral regions of the embryo (Ferguson and Anderson 1992a,b; Wharton et al. 1993). In this model, peak Dpp activity specifies the dorsal-most cell type lamnioserosa), whereas lower levels of activity specify the dorsal ectoderm. It is likely that small amounts of Dpp also diffuse into the lateral zone to influence patterning in the neuroectoderm.

As the level of $d p p$ mRNA appears uniform throughout the dorsal zone, it has been speculated that a posttranscriptional mechanism is responsible for establishing graded Dpp activity. The short gastrulation $|\operatorname{sog}\rangle$ gene may contribute to generating such a Dpp activity gradient. $s o g$ is expressed in broad lateral stripes abutting $d p p$-expressing dorsal cells (François et al. 1994) and functions nonautonomously to pattern the dorsal region of the embryo (Zusman et al. 1988; Ray et al. 1991; Ferguson and Anderson 1992b; Wharton ct al. 1993; François et al. 1994). The sog product is a likely secreted protein (François et al. 1994) that may diffuse into the dorsal region to antagonize Dpp signaling (Zusman et al. 1988; Ferguson and Anderson 1992b; Wharton et al. 1993; François et al. 1994). It is unclear, however, whether $s o g$ also functions locally to block $d p p$ activity in the neuroectoderm itself. This question is of particular interest as chordin, the Xenopus homolog of sog, prevents the Dpp homolog BMP-4 from suppressing neurogenesis during early frog development (Sasai et al. 1995; Wilson and Hemmati-Brevanlou 1995). As Chordin has been shown recently to bind BMP-4 with high affinity (Picolla et al. 1996), the mechanism of Chordin/Sog function may be to bind and sequester BMP-4/Dpp in an inactive form.

In this paper we investigatc the role of $\operatorname{sog}$ in the lateral neuroectoderm of the Drosophila blastoderm embryo. We show that sog can block two distinct $d p p$ functions (i.e., suppression of neural genes and activation of ZDG genes) in both dorsal and lateral regions of the early blastoderm embryo. We show that these two dpp functions have different thresholds for Dpp activity as considerably less Dpp activity is required for neural suppression than for dorsalization. Epistasis experiments indicate that sog functions primarily or exclusively to block Dpp signaling. We also provide evidence that Sog may act over long distances in a dose-dependent fashion to determine the limits of gene expression domains. These data support the view that $\operatorname{sog}$ acts locally in the neuroectoderm to block Dpp autoactivation, thereby providing a permissive condition for initiating neurogenesis. In addition, Sog behaves as a long-range diffusible morpho- gen to define distinct thresholds of Dpp activity in neighboring regions of the blastoderm embryo.

\section{Results}

Dpp suppresses neurogenesis in dorsal cells of early embryos

To assess the role of $d p p$ in regulating neurogenesis, we first examined the effect of this signaling pathway on neurogenesis in dorsal cells where $d p p$ is known to play a critical role in cell fate determination. The dorsal cuticle of embryos lacking $d p p$ activity appears ventralized (Irish and Gelbart 1987), leading to the inference that $d p p$ functions normally to suppress lateral cell fates dorsally. This inference is tenuous, however, as the number of differentiated neurons is reduced, not expanded in late $d p p^{-}$mutant embryos (B. Biehs and E. Bier, unpubl.). In early gastrulating $\mathrm{dpp}^{-}$mutants, on the other hand, we observed dorsal expression of neuroectodermal markers such as thick veins $(t k v)$, which encodes a type I Dpp receptor, and lethal of scute (1'sc) (Fig. 1, cf. B and E with $\mathrm{A}$ and D). Similarly, neuroblasts visualized by markers such as scratch (scrt) and snail (sna) subsequently form ectopically in the dorsal region of gastrulating $d p p^{-} \mathrm{em}^{-}$ bryos (Wharton et al. 1996; B. Biehs, unpubl.). Consistent with $d p p$ acting early to suppress initiation of neurogenesis, ectopic expression of the proneural gene l'sc is first detectable in dorsal cells of late blastoderm stage $d p p^{-}$ embryos (see arrow in Fig. 4G). Paradoxically, the increased number of neuroblasts in $d p p^{-}$mutants do not generate a hypertrophied differentiated nervous system. Thus, $d p p^{-}$mutants may lack a late positive role for $d p p$ in neuronal maturation or may hyperactivate pathways functioning to inhibit subsequent steps in neurogenesis.

sog prevents Dpp from suppressing neurogenesis laterally in the blastoderm embryo

Superficially, sog does not appear to play a major role in early neurogenesis as defects in nervous system formation in $\operatorname{sog}^{-}$mutant embryos are detectable only during midgastrulation and are relatively mild (François et al. 1994). Because $s$ g behaves genetically as a uniquely potent suppressor of Dpp signaling (Ferguson and Anderson $1992 \mathrm{~b}$ |, we reasoned that it might be possible to exacerbate defects in $\operatorname{sog}^{-}$embryos by increasing the level of Dpp. Thus, we examined expression of various neuroectodermal markers in sensitized $\operatorname{sog}^{-}$embryos that have twice the normal dose of $d p p\left\{s o g^{-}\right.$; Dpdpp: i.e., $0 \times \operatorname{sog}$ and $4 \times d p p \mid$. The earliest neuroectodermal marker affected in $\operatorname{sog}^{-}$; Dpdpp embryos is rhomboid (rho), which is expressed in lateral stripes $8-10$ cells wide in wildtype blastoderm embryos (Bier et al. 1990). Lateral rho expression is initiated normally in $\operatorname{sog}^{-}$; Dpdpp embryos, but rapidly narrows to stripes $4-6$ cells across (Fig. 2 , cf. B with A). Similarly, the lateral extent of l'sc expression is reduced in early gastrulating $\operatorname{sog}^{-}$; Dpdpp embryos (Fig. 2E) relative to wild type (Fig. 2D), resulting in the subsequent loss of the outer two rows of scrt- 

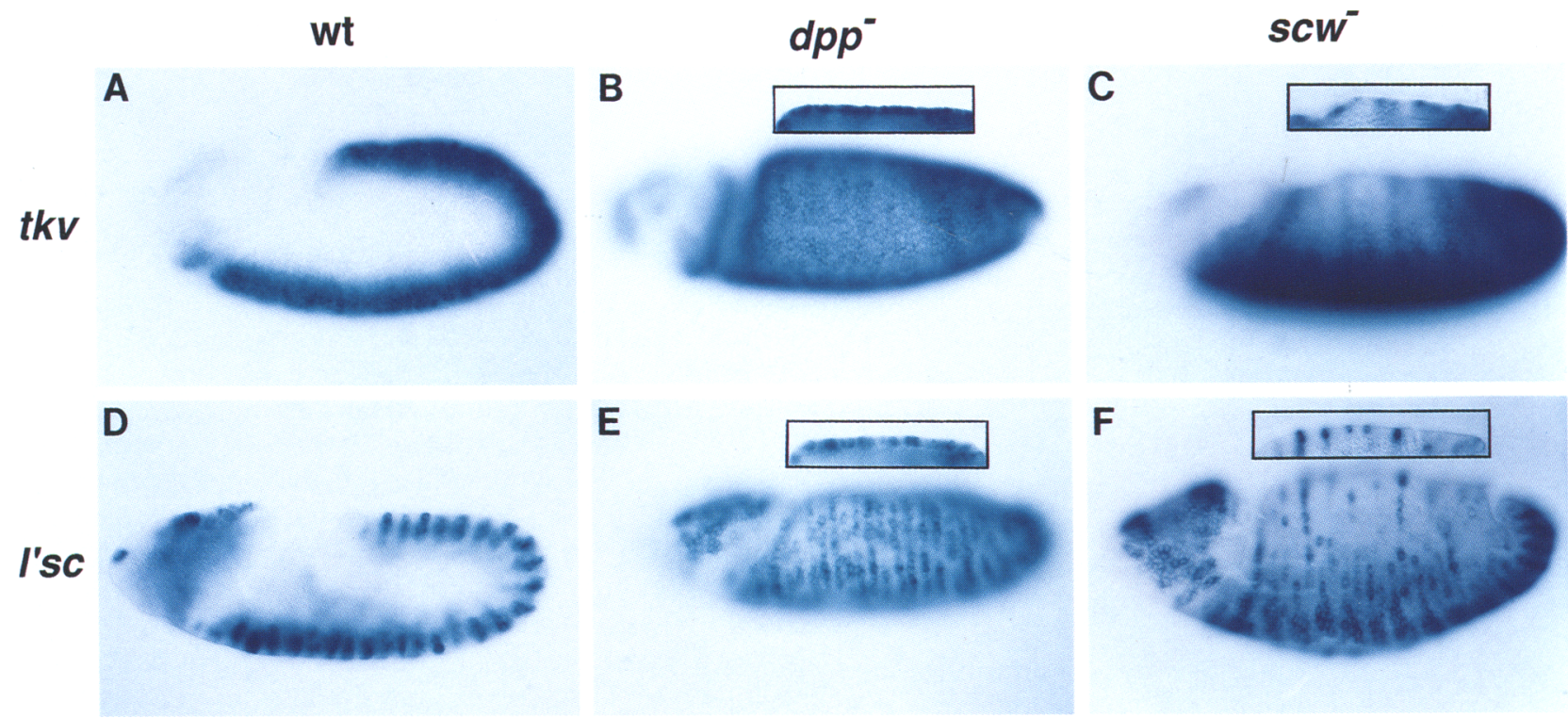

Figure 1. dpp suppresses neurogenesis in the dorsal region of the embryo. Expression of the neuroectodermal markers $t k v$ and $l$ 'sc in wild-type (wt) and mutant embryos. Lateral views of embryos are shown with anterior to the left and dorsal at the top in this and subsequent figures unless indicated otherwise. Insets in $B, C, E$, and $F$ show sagittal sections near the dorsal midline. $(A) t k v$ expression is restricted to the neuroectoderm in an early wild-type germ-band extending embryo. $(B) t k v$ expression in a homozygous $d p p^{\text {hin }}$ null mutant $\left(d p p^{-}\right)$embryo occupies the entire dorsal ectoderm as well as the neuroectoderm. Because $d p p^{-}$embryos do not germ-band extend normally, they appear younger than comparably staged wild-type embryos. $(C)$ As in wild type, tkv expression is largely suppressed in the dorsal ectoderm of a homozygous $s c w^{512}$ mutant $\left(s c w^{-}\right)$embryo. $s c w^{-}$mutants, like $d p p^{-}$embryos, do not germ-band extend normally. $(D)$ l'sc expression is restricted to the neuroectoderm in an early wild-type germ-band extending embryo. (E) l'sc expression in a homozygous $d p p^{-}$embryo extends into the dorsal ectoderm. Ectopic dorsal expression of $l^{\prime} s c$ in $d p p^{-}$mutants is first observed during the late blastoderm stage (see Fig. 4G). (F) l'sc expression is largely suppressed as normal in the dorsal ectoderm of a $s c w^{-}$embryo.

expressing neuroblasts (Fig. 2, cf. $\operatorname{sog}^{-}$in $\mathrm{H}$ with G). As these phenotypes are only observed in $\operatorname{sog}^{-}$; Dpdpp double mutant embryos and not in either single mutant, other genes must normally function in parallel with $s o g$ to block the action of Dpp (see below). It is also possible to overcome the protective function of sog in the neuroectoderm by forcing strong ubiquitous expression of $d p p$ in a $\operatorname{sog}^{+}$background. Thus, heat induction of blastoderm embryos carrying eight copies of a HS- $d p p$ construct $(8 \times$ HS- $d p p)$ reduces lateral expression of $r h o$ to stripes 5-7 cells wide (Fig. 2C). Such ubiquitous $d p p$ expression also narrows the subsequent expression of l'sc (data not shown), $t k v$ (data not shown), and scrt (Fig. 2I) during germ-band extension. The neuronal loss phenotype of HS-dpp embryos is enhanced in $\operatorname{sog}^{-}$embryos that lack all but the ventral-most neural progenitors (Fig. $2 F$ ). These early neuronal loss phenotypes are consistent with the moderate loss of ventral denticles observed in sog $^{-}$mutant cuticles (Wieschaus et al. 1984; Zusman et al. 1988) and with the exacerbation of this cuticular phenotype by extra copies of $d p p$ (Ferguson and Anderson 1992b).

$d p p$ continues to exert a neural suppressive function during early gastrulation as HS- $d p p$ induction during germ-band extension causes a significant reduction in neuroblast number (data not shown). As the D-V axis is well established by germ-band extension, the continued neural suppressive activity of $d p p$ during this stage suggests that Dpp signaling functions by suppressing neurogenesis per se rather than acting indirectly by suppressing the establishment of ventral positional values. Cumulatively, the above results show that sog acts in concert with other genes during the blastoderm stage to prevent Dpp signaling from suppressing neurogenesis in lateral cells.

\section{Dpp can activate expression of dorsal domain genes in the neuroectoderm}

In addition to suppressing expression of neuroectodermal genes in the dorsal region, Dpp also plays an active dorsalizing role by maintaining expression of ZDG genes such as zen. In $d p p^{-}$embryos, expression of zen is initiated correctly but then disappears rapidly (Ray et al. 1991). Therefore, we asked whether $d p p$ could activate expression of dorsal markers in the early neuroectoderm of $s o g^{-}$; Dpdpp mutants. Surprisingly, we observed that expression of $d p p$ itself was induced throughout the neuroectoderm in $\operatorname{sog}^{-}$; Dpdpp embryos (Fig 3, cf. B with A). This provides the first evidence that $d p p$ is capable of autoactivating during early embryogenesis. The ventral limit of $d p p$ expression (blue) is sharp and lies one cell diameter within the sna (brown)-expressing mesoderm (Fig. 3B; inset). Double label experiments with a combination of rho and $d p p$ probes revealed that $d p p$ autoac- 

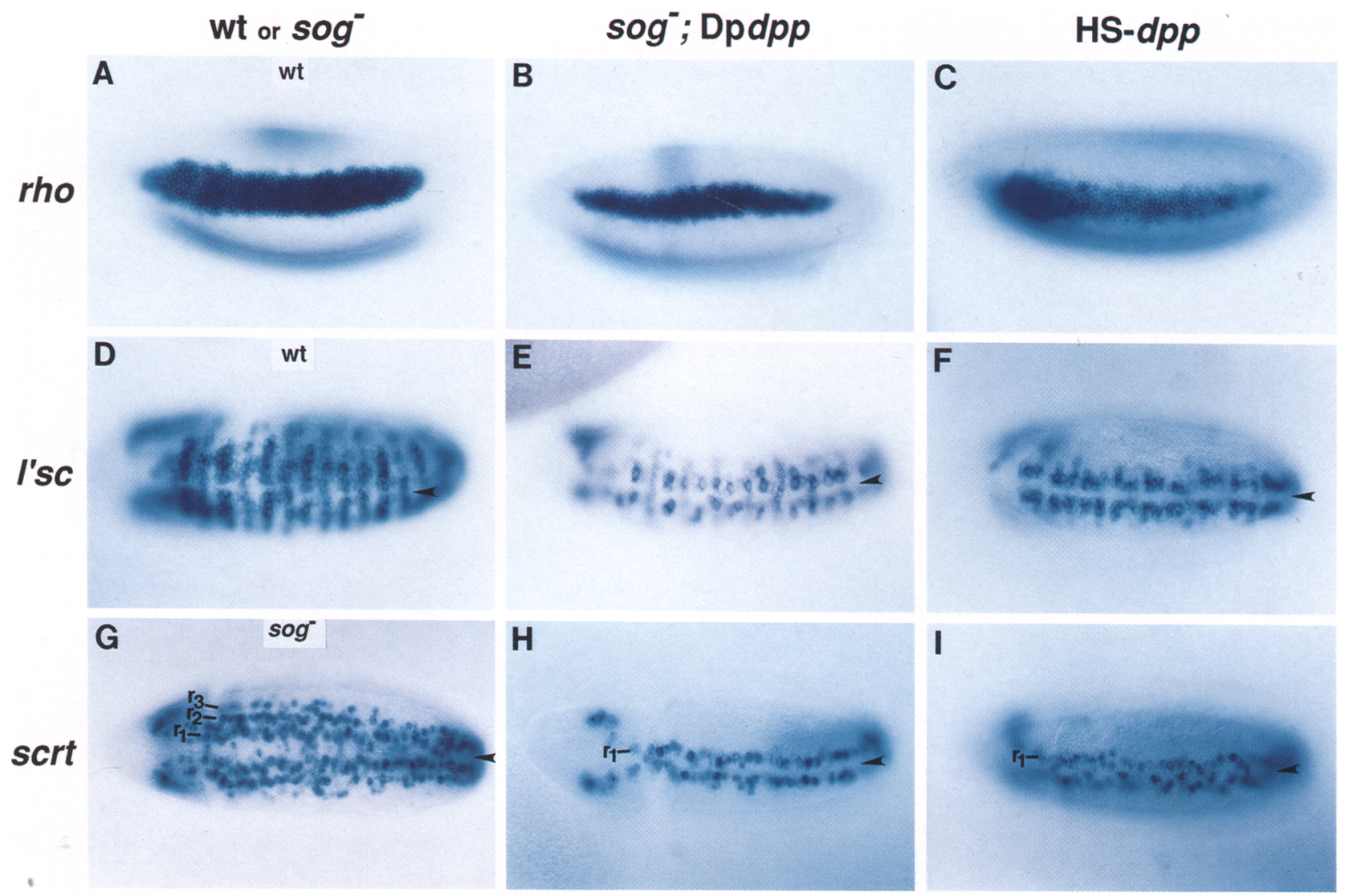

Figure 2. Dpp can suppress neurogenesis in the lateral region of the embryo. Expression of neuroectodermal markers during the early precellular blastoderm stage (rho; $A-C$ ), early gastrulation before neuroblast delamination (1'sc; $D-F$ ), and late germ-band extension (scrt; $G-I$ ). Embryos in $D-I$ are viewed from a ventral perspective with anterior to the left. In $D-F$ surface views are shown and in $G-I$ the focal plane is subectodermal. The ventral midline in $D-I$ is denoted by an arrowhead. $(A)$ rho expression in lateral stripes is 8-10 cells wide in a wild-type precellular blastoderm embryo. $(B)$ The dorsal extent of the lateral rho stripes is reduced by about four cells in $\operatorname{sog}^{\mathrm{U} 2} / \mathrm{Y}$; Dpdpp/Dpdpp $\left(\operatorname{sog}^{-}\right.$; Dpdpp) embryos. (C) Lateral rho stripes are similarly narrowed in $\operatorname{sog}^{+}$embryos carrying eight copies of the heat shock $d p p$ construct $(8 \times$ HS- $d p p)$ after a 20-min heat shock and 30-min of recovery. $(D)$ Wild-type expression of 1 'sc in lateral stripes in an early gastrulating embryo. (E) The dorsal extent of $1^{\prime} s c$ expression is significantly reduced in $s o g^{-}$; Dpdpp embryos. (F) l'sc expression in a $s o g^{\mathrm{U}} / \mathrm{Y}$ embryo carrying four copies of the HS-dpp construct $\left(\operatorname{sog}^{-} ; 4 \times\right.$ HS-dpp) that was heat shocked for 30 min during the blastoderm stage and allowed to develop until midgastrulation. Embryos of this kind have the most pronounced reduction of l'sc expression we observed. $(G)$ scrt expression in $\mathrm{S}_{1}$ and early $\mathrm{S}_{2}$ neuroblasts in a sog $/ \mathrm{Y}$ hemizygous embryo. The number of neuroblasts expressing scrt is mildly reduced. Typically, the thoracic and most anterior abdominal segments have the full three rows $\left(r_{1}-r_{3}\right)$ of $S_{1}$ neuroblasts, the middle abdominal segments have the two inner rows $\left(r_{1}, r_{2}\right)$, and the posterior segments have only the inner $r_{1}$ row of neuroblasts [not visible in this focal plane, see Fig. 1H in François et al. (1994)]. (H) scrt expression in neuroblasts is reduced to the inner $\mathrm{r}_{1}$ row along the entire length of a $\operatorname{sog}^{-}$; Dpdpp embryo. $(I)$ A similar strong reduction in neuroblasts is observed in $\operatorname{sog}^{+} ; 8 \times$ HS- $d p p$ embryos that were heat shocked only $13 \mathrm{~min}$ and then allowed to develop until germ-band extension. With this minimal heat induction there is no detectable ectopic expression of dorsal markers in the neuroectoderm (data not shown).

tivation precedes or is contemporaneous with the narrowing of lateral rho expression in $\operatorname{sog}^{-}$; Dpdpp embryos (Fig. 2B). We also observed ectopic expression of zen (Fig. 3 , cf. D with C) and tld (data not shown) in the neuroectoderm of $\operatorname{sog}^{-}$; Dpdpp embryos. As in the case of neural suppression, dpp autoactivation and misexpression of zen and tld were not observed in either $\operatorname{sog}^{-}$or Dpdpp single mutant embryos (data not shown). These data reveal that Dpp signaling, if unopposed by sog, can invade the neuroectoderm by a chain of diffusion followed by autoactivation. Ubiquitous expression of $d p p$ in a $\operatorname{sog}^{+}$background via HS-dpp also leads to ectopic activation of zen expression in parts of the neuroectoderm (Fig. 3E) and mesoderm (Fig. 3E, inset; see also Fig. $5 \mathrm{D}, \mathrm{E}$ below). The effect of ubiquitous $d p p$ expression is enhanced greatly in a $\operatorname{sog}^{-}$background (Fig. 3F), resulting in zen expression throughout the entire trunk neuroectoderm and mesoderm (see also Fig. $5 \mathrm{~F}$ below).

Neural suppression is induced by a lower threshold of Dpp activity than dorsalization

A striking feature of the data presented above is that Dpp-mediated neural suppression and dorsalization appear to have markedly different thresholds for Dpp ac- 
Figure 3. sog opposes Dpp autoactivation in the neuroectoderm. Expression of the early dorsal markers $d p p(A, B, G, H)$ and zen $(C-F)$ in the neuroectoderm is opposed by sog. Double label in situ hybridizations (O'Neill and Bier 1994) were performed with digoxigenin-labeled probes /visualized by a blue alkaline phosphatase reaction product) and biotin-labeled probes (visualized by a brown peroxidase reaction product). (A) $d p p$ (blue) and sna (brown) expression in a wild-type (wt) blastoderm stage embryo. sna expression marks the prospective mesoderm. $(B) d p p$ and sna expression in a $\operatorname{sog}^{-}$; Dpdpp embryo. $d p p$ is expressed ectopically throughout the neuroectoderm and descends into the dorsalmost row of mesoderm cells (small arrow; arrowhead in inset). The large arrow indicates a stripe of elevated ectopic $d p p$ expression. $(C)$ zen (blue) and sna (brown) expression in a wild-type embryo. (D) $z e n$ and sna expression in a $s o g ; D p d p p$ embryo. zen is expressed ectopically in a large portion of the neuroectoderm and extends ventrally into the most dorsal row of mesoderm cells (arrow; arrowhead in inset). (E) zen and sna expression in a $\operatorname{sog}^{+} ; 8 \times$ HS- $d p p$ embryo after $30 \mathrm{~min}$ of heat shock and $30 \mathrm{~min}$ of recovery. zen expression extends ventrally four to six cells in the central region of the neuroectoderm and encircles the embryo in vertical stripes at the head and tail (inset shows a cross-sectional focal plane in the mesoderm). The ventral and lateral portions of the neuroectoderm do not express zen. Identical phenotypes are observed in $\operatorname{sog}^{+} ; 4 \times$ HS- $d p p$ embryos after $30 \mathrm{~min}$ of heat shock and $30 \mathrm{~min}$ of recovery. $(F)$ zen and sna expression in a $\operatorname{sog}^{-} ; 4 \times$ HS- $d p p$ embryo after 30 min of heat shock and 30 min of recovery. zen expression occupies the entire trunk neuroectoderm and mesoderm in these embryos. In sibling $\operatorname{sog}^{-} /+; 4 \times$ HS-dpp embryos the pattern of zen expression in the neuroectoderm is similar to that shown in $E$. $(G) d p p$ (blue) and sna (brown) expression in a wild-type germ-band extended embryo. Note that the ventral limit of the $d p p$ domain precisely abuts the central nervous system neuroectoderm at this stage (arrow). $(H) d p p$ and $s n a$ in a $\operatorname{sog}^{-} / \mathrm{Y}$ embryo derived from mothers heterozygous for a loss of function dorsal allele $\left(d l^{-} /+\right)$. There is significant ectopic $d p p$ expression in the central nervous system. Ectopic $d p p$ expression also can be observed during the cellular blastoderm stage when the limit of $d p p$ expression is shifted ventrally by four to six cells and clusters of six to eight $d p p$-expressing cells are found near the mesoderm in the region of the cephalic furrow (this area is indicated by the large arrow in $B ; \mathrm{B}$. Biehs, unpubl.).

tivity. In all cases examined, much less Dpp activity was required to suppress expression of neuroectodermal genes than was required to activate dorsal markers. For example, brief submaximal heat induction of $\operatorname{sog}^{+}$; HS$d p p$ blastoderm embryos led to nearly maximal suppression of l'sc, scrt (Fig. 2I), or sna expression during germband extension. Under these conditions, however, there is no detectable ectopic expression of zen in the neuroectoderm (data not shown). In contrast, maximal HS- $d p p$ induction is required to induce neuroectodermal expression of zen (the most sensitive dorsal marker) in a sog $^{-}$ background (Fig. 3F). Similarly, in dorsolateral cells of strongly induced $\operatorname{sog}^{+}$; HS- $d p p$ blastoderm embryos, lat- eral rho expression is suppressed (Fig. 2C) without inducing ectopic zen expression (Fig. 3E). More Dpp activity is also required to activate zen than to suppress neural markers in dorsal cells. Thus, as in $d p p^{-}$embryos, zen expression is not maintained in $\mathrm{scW}^{-}$mutants (Ray et al. 1991) or in heat-shocked HS-sog embryos (B. Biehs, unpubl.; see below for description of the HS-sog phenotype). In contrast, neural suppression remains largely intact in the dorsal region of $\mathrm{scW}^{-}$embryos (Fig. 1; cf. C and $F$ with $B$ and $E$ ) or HS-sog embryos (data not shown). These observations are also consistent with weak neural suppression but lack of ectopic dorsal gene expression in the neuroectoderm of $\operatorname{sog}^{-}$single mutants. 
Dorsal acts in parallel with sog to repress dpp expression in the neuroectoderm

During establishment of primary D-V domains of zygotic gene expression, maternally supplied Dorsal actively represses expression of $d p p$ and other ZDG genes in lateral and ventral regions of the blastoderm embryo (Huang et al. 1993, 1995; Jiang et al. 1993). Thus, dorsal seemed a likely candidate for one of the genes acting in concert with sog to block $d p p$ autoactivation in the neuroectoderm. Consistent with this expectation, $d p p$ is expressed ectopically throughout the central nervous system of $\operatorname{sog}^{-}$germ-band extended embryos, which are derived from mothers with only one functional copy of dorsal $\left(\mathrm{dl}^{-} /+\right.$; Fig. $\left.3 \mathrm{H}\right)$. There are also earlier defects in blastoderm stage embryos of this genotype including a shift in the ventral limit of $d p p$ expression by aproximately four cells and small patches of ectopic $d p p$ expression where the neuroectoderm/mesoderm boundary intersects the cephalic furrow (data not shown). Similar blastoderm stage phenotypes are observed in embryos with four copies of $d p p$ derived from $d l^{-} /+$mothers. Thus, maternal Dorsal is likely to be one of the genetic elements acting in parallel with sog to prevent Dpp from autoactivating in the neuroectoderm.

\section{sog functions upstream of $\mathrm{dpp}$}

The observation that strong ubiquitous $d p p$ expression in a $\operatorname{sog}^{+}$background leads to phenotypes similar to those found in $\operatorname{sog}^{-}$; Dpdpp embryos is consistent with models in which sog functions primarily or exclusively to block Dpp signaling. To determine the epistatic relationship between $s o g$ and $d p p$ in greater detail, we examined expression of several marker genes in $s o g^{-} ; d p p$ double mutant embryos (Fig. 4). The most informative markers for this purpose are misexpressed in opposite ways in $\operatorname{sog}^{-}$versus $d p p^{-}$mutants. For example, the dorsal stripe of rho expression, which gives rise to the amnioserosa in wild-type embryos (Figs. 4A and 5A), is expanded in $\operatorname{sog}^{-}$mutants to include nearly the full dorsal region (Figs. $4 \mathrm{~B}$ and $5 \mathrm{C}$ ), but is entirely lacking in $d p p^{-}$mutants (Fig. 4D; François et al. 1994). In $s o g^{-} ; d p p^{-}$double mutant embryos, dorsal rho expression is lost (Fig. 4E) as it is in $d p p^{-}$single mutants (Fig. 4D). Similarly, expression of race, another early amnioserosa marker (Tatei et al. 1995), is expanded in $\operatorname{sog}^{-}$ embryos, but is absent in both $d p p^{-}$single mutants and $s o g^{-} ; d p p^{-}$double mutants (data not shown). Conversely, expression of neural markers such as l'sc, scrt, $s n a$, or $t k v$ expands into the dorsal region of gastrulating $d p p^{--}$mutant embryos (e.g., Fig. 1B,E), but is either narrowed (e.g., scrt and sna; see Fig. 2G) or unaffected (e.g., $l^{\prime} s c$ and $\left.t k v\right)$ in $\operatorname{sog}^{-}$mutants. In $s o g^{-} ; d p p^{-}$double mutant embryos, expression of l'sc (Fig. $4 \mathrm{H}$ ) and other neural marker genes (data not shown) is indistinguishable from that in $d p p^{-}$single mutants. Furthermore, we found no differences between the expression of the dorsal markers $d p p$, zen, and $t l d$ and the ventral ectodermal marker ot $d$ in $s g^{-} ; d p p^{-}$double mutant embryos versus $d p p^{-}$single mutants (data not shown).
We also addressed the epistasis between $s o g$ and $d p p$ by comparing the effects of ectopically expressing sog in wild-type versus $d p p^{-}$mutant backgrounds. The motivation for these experiments is that if $\operatorname{sog}$ acted on a pathway independent of the Dpp pathway, overexpressing sog might aggravate the $d p p^{-}$mutant phenotype.

We first characterized the effect of ubiquitous sog misexpression in a wild-type background using a line of flies carrying eight copies of a HS-sog construct ( $8 \times$ HS-sog). When $8 \times$ HS-sog blastoderm embryos were heat shocked, we observed phenotypes typical of moderate loss of function ZDG mutants. For example, in $8 \times \mathrm{HS}$-sog embryos, rho expression is eliminated in the dorsal stripe (Fig. 4F), but is unaffected in the head stripe and lateral stripes, which are independent of $d p p$ activity (see Fig. 4D). This HS- $s o g$ phenotype is opposite to that of HS- $d p p$ embryos in which the dorsal rho stripe expands beyond the limits of the normal dorsal domain (Fig. 4C). In $8 \times$ HS-sog embryos, as in $d p p^{-}$mutants, we observed that $t l d$ expression was normal and that zen expression was initiated normally but not maintained (data not shown). Ectopic sog expression also relieves neural suppression dorsally resulting in ectopic dorsal stripes of l'sc expression (Fig. 4I). Such early $8 \times$ HS-sog embryos subsequently give rise to ectopic scrt-expressing neuroblasts /data not shown). The extent of ectopic l'sc and scrt expression in $8 \times$ HS-sog embryos is similar to that in $s c W^{-}$mutants, which is less extreme than in $d p p^{-}$null mutants.

To test whether ectopic sog expression enhanced the $d p p^{-}$phenotype, we conducted parallel HS-sog inductions in $d p p^{-}$embryos carrying four copies of the HS-sog transgene $(4 \times$ HS-sog). We compared expression of dorsal markers (rho, zen, and tld) and neural markers ( 1 'sc and scrt) in $4 \times \mathrm{HS}-s o g$; $d p p^{+}$embryos to that in $4 \times$ HS-sog; $d p p^{-}$embryos. As in the case of the $8 \times$ HSsog line, we observed highly penetrant loss of the dorsal rho stripe and ectopic dorsal expression of l'sc and scrt in $4 \times$ HS-sog; $d p p^{+}$embryos. On the other hand, sibling $4 \times$ HS-sog; $d p p^{-}$embryos had a phenotype indistinguishable from $d p p^{-}$single mutants (data not shown). Similarly, dorsal expression of $t l d$ is normal in $4 \times$ HS$s o g$; $d p p^{-}$double mutants and expression of zen is initiated normally and then fades with the same time course as observed in $d p p^{-}$single mutants. Thus, although ectopic sog expression in a wild-type background generates a phenotype comparable to that of $\mathrm{scw}^{-} \mathrm{mu}-$ tants, identical sog misexpression in a $d p p^{-}$background has no detectable effect.

Sog acts over a long distance in a dose-dependent fashion

Previous studies indicated that sog functions nonautonomously to influence cell fates over significant distances (Zusman et al. 1988; François et al. 1994). It was also observed that reducing the gene dose of sog could rescue the lethality of weak ZDG mutants (Ferguson and Anderson 1992b; Wharton et al. 1993; François et al. 1994). To determine whether the dose of sog can influ- 

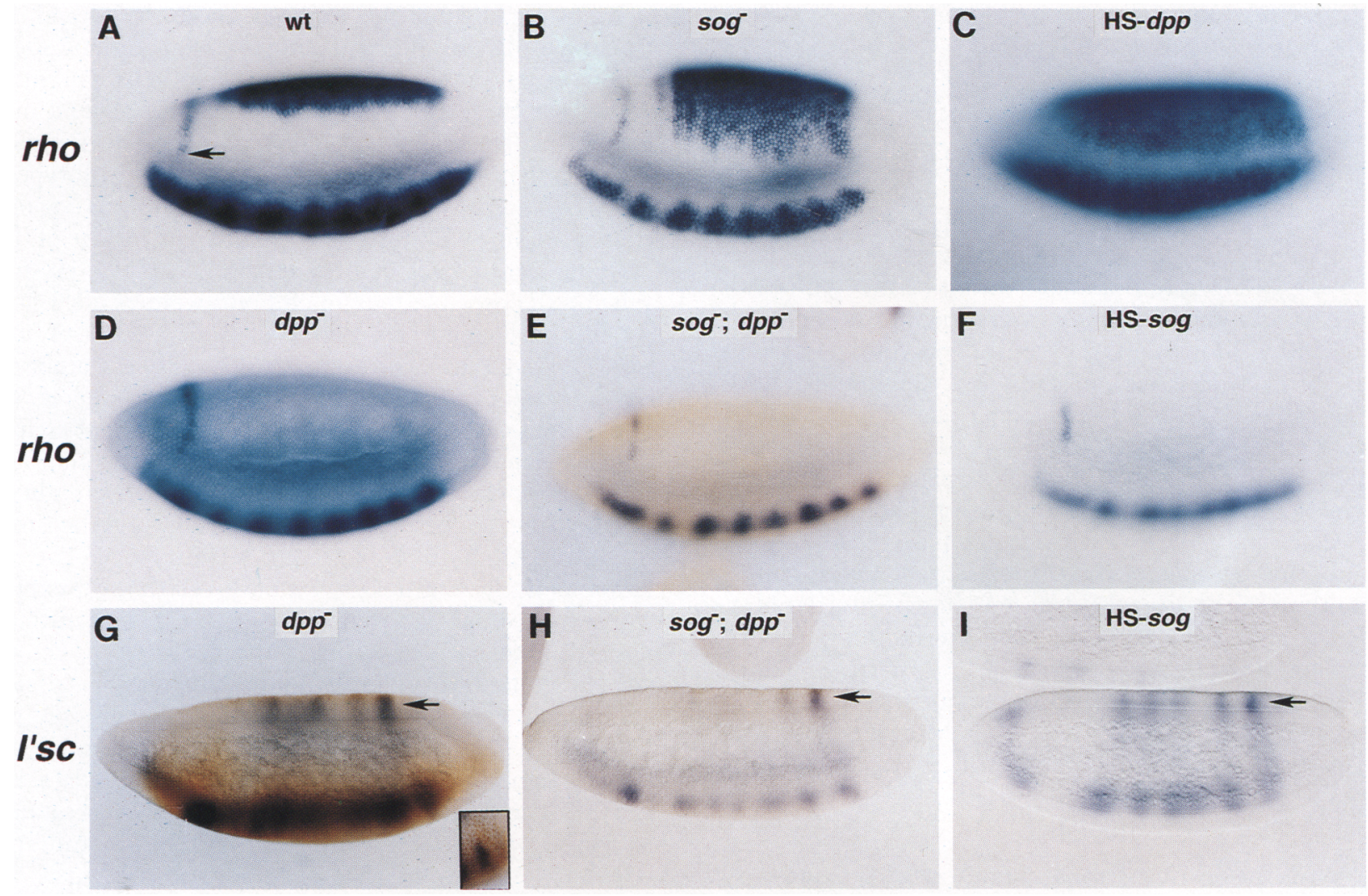

Figure 4. $d p p$ is epistatic over sog. Expression of rho $(A-F)$ and $l$ 'sc $(G-I)$ in sog and $d p p$ mutant embryos is consistent with Sog functioning primarily or exclusively to block Dpp signaling. Surface views of embryos are shown in $A-F$ and cross-sectional views are shown in $G-I$. $(A)$ rho expression in a wild-type embryo. The arrow indicates the head stripe that serves as a reference point. Note that the dorsal rho stripe does not extend as far ventrally as the head stripe. (B) rho expression in a sog ${ }^{2} / Y$ RNA null mutant embryo. This embryo was double labeled with a biotin-sog probe to score for the $\operatorname{sog}^{-}$genotype. Note that the dorsal rho stripe is expanded such that the ventral border of this domain coincides with the ventral extent of the head stripe (cf. wild-type embryo in $A$; see also Figs. $5 \mathrm{~A}, \mathrm{C}$ for dorsal views of the same genotypes). $(C)$ rho expression in a heat-shocked $8 \times \mathrm{HS}$-dpp embryo. Embryos were heat shocked for $30 \mathrm{~min}$ at $38^{\circ} \mathrm{C}$, allowed to recover at room temperature for another $30 \mathrm{~min}$, and then fixed for analysis. The extreme ventral expansion of the dorsal rho stripe can be gauged by the small gap (four to six cells) separating dorsal and lateral rho stripes. (D) rho expression in a Df $d p p$ embryo. This embryo was double hybridized with a digoxigenin labeled $d p p$ probe to identify unambiguously mutant embryos (i.e., those embryos not expressing $d p p$ ). Identical phenotypes were observed in embryos homozygous for two other $d p p^{\text {hin }}$ null point alleles. The dorsal rho stripe is entirely lacking at all developmental stages in $d p p^{-}$embryos. rho expression in the head stripe, which is independent of Dpp activity, and in lateral stripes (out of focus) is normal, however. $(E)$ rho expression in a $s o g^{\mathrm{U}} / \mathrm{Y} ; d p p^{\text {hin }} / d p p^{\text {hin }}$ double mutant embryo $\left(s \operatorname{sog}^{-} ; d p p^{-}\right)$. This embryo was double hybridized with a biotin-sog probe. The phenotype of $s o g^{-}$; $d p p^{-}$embryos is indistinguishable from that of $d p p^{-}$single mutants (e.g., cf. $\left.D\right)$. $(F)$ rho expression in $8 \times$ HS-sog embryos is very similar to that observed in $d p p^{-}$mutants. Embryos were heat shocked for $30 \mathrm{~min}$ at $38^{\circ} \mathrm{C}$, allowed to recover at room temperature for another $30 \mathrm{~min}$, and then fixed for analysis. Similar, although weaker, phenotypes were also observed by expressing sog ectopically in dorsal blastoderm cells by means of a UAS-sog transgene activated by zen-GAL4 or $d p p$-GAL4 driver constructs (data not shown). (G) l'sc expression in a $s o g^{+} ; d p p^{-}$sibling of the $s o g^{-} ; d p p^{-}$double mutant embryo shown in $H$. The arrow points to abnormal dorsal expression of $l^{\prime} s c$. In wild-type or $\operatorname{sog}^{-}$embryos this region is devoid of staining. The inset shows a lateral surface view of the same embryo with strong biotin-sog staining revealing that this embryo is $\operatorname{sog}^{+} .(H) l^{\prime} s c$ expression in a $s o g^{-}$; $d p p^{-}$double mutant embryo. This embryo was double hybridized with a biotin-sog probe. The phenotype of such embryos is indistinguishable from that of $d p p^{-}$single mutants (e.g., cf. G). The arrow points to abnormal dorsal expression of l'sc. (I) l'sc expression in a $8 \times$ HS-sog embryo. Ectopic l'sc expression in the dorsal region of this embryo is similar to that observed in $d p p^{-}$, in $4 \times \mathrm{HS}_{-} s \mathrm{~g}$; $d p p^{+}$, and in $4 \times$ HS-sog; $d p p^{-}$embryos (data not shown). The arrow indicates abnormal dorsal expression of l'sc.

ence the extent of gene expression domains, we compared the width of the dorsal rho stripe in embryos carrying two doses of $\operatorname{sog}$ (i.e., wild type), one dose of $\operatorname{sog}$ (i.e., $\operatorname{sog}^{-} /+1$, and zero doses of $\operatorname{sog}\left(\right.$ i.e., $\operatorname{sog}^{-} /$Y). In wild-type $(2 \times$ sog) embryos, dorsal rho expression is initiated as a solid domain $\sim 15$ cells wide and then narrows to an irregular stripe $10-13$ cells across at maximal extent in late blastoderm embryos (Fig. 5A). The dorsal tho stripe is expanded in $\operatorname{sog}^{-} /+(1 \times$ sog $)$ embryos, differing most from wild type in late blastoderm embryos (Fig. 5B) when it is typically $17-20$ cells wide. The width of the dorsal rho stripe is broadened similarly in $2 \times$ sog em- 


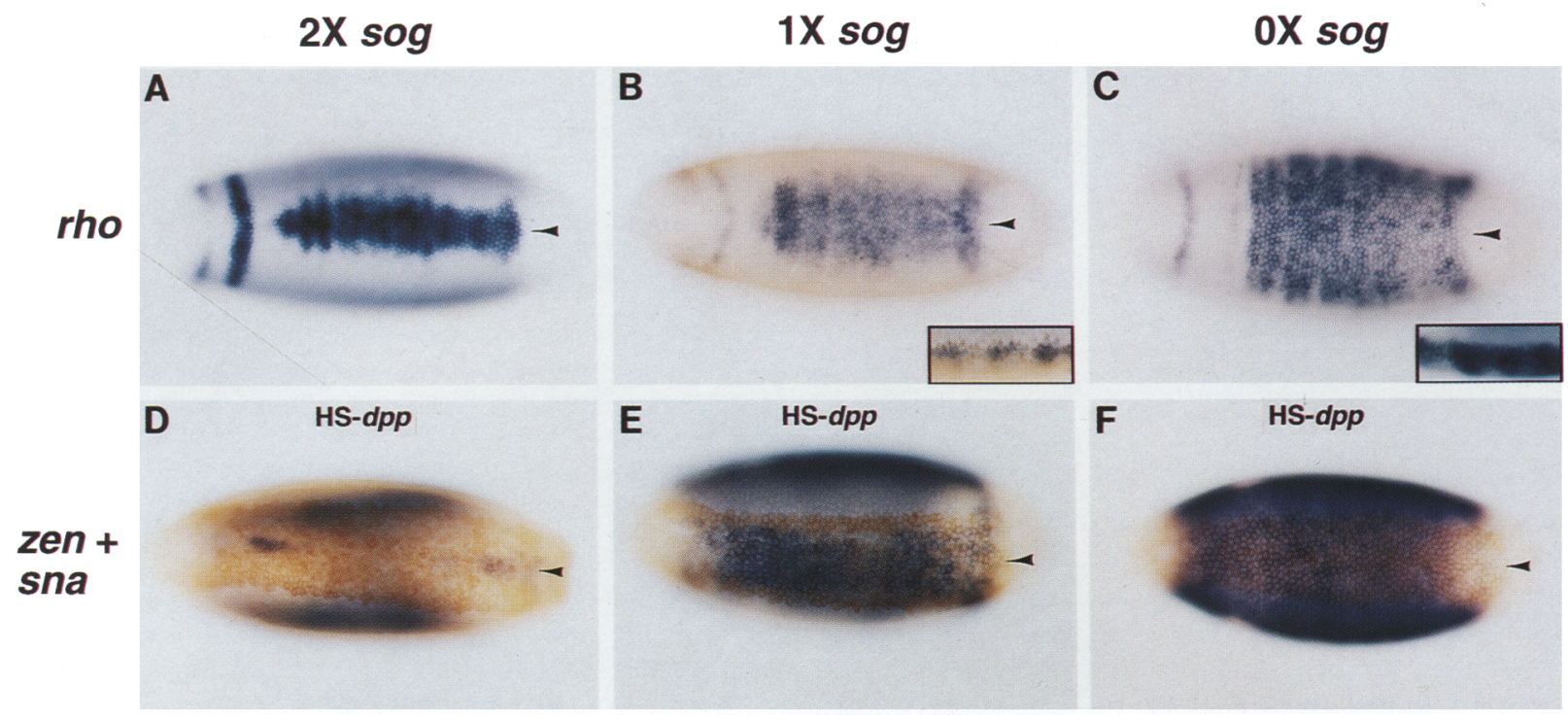

Figure 5. sog exerts a long range dose-dependent influence on gene expression. Wild-type embryos carrying two functional copies of $\operatorname{sog}(2 \times \operatorname{sog})$, embryos heterozygous for a sog null allele $(1 \times \operatorname{sog})$, and embryos lacking all sog function $(0 \times$ sog) express marker genes in a dose-dependent fashion. Embryos in $A-C$ were hybridized with a digoxigenin-labeled rho antisense RNA probe and $(B, C)$ with a biotin-labeled sog probe. Embryos are viewed dorsal side up with anterior facing to the left. The arrowhead in $A-C$ denotes the dorsal midline. Embryos in $D-F$ were double hybridized with a digoxigenin-labeled zen probe and a biotin-labeled sna probe and are viewed ventral side up with anterior facing to the left. The arrowhead in $D-F$ denotes the ventral midline. $(A)$ Dorsal $r$ ho expression in a wild-type embryo $(2 \times \operatorname{sog})$ is $\sim 10-13$ cells in maximum width. $(B)$ Dorsal $r$ ho expression in a $\operatorname{sog} \mathrm{U} 2 /+$ embryo $(1 \times$ sog $)$ increases to 17-20 cells across. This embryo can be scored as $\operatorname{sog}^{-} /+$as it expresses $s o g$ weakly (see inset), and because in parallel hybridizations of wild-type embryos such an expansion of the dorsal rho stripe is not observed. The dorsal rho stripe is expanded comparably in each of the three $\operatorname{sog}$ alleles we tested (i.e., $\operatorname{sog}^{\mathrm{P} 1}, \operatorname{sog}^{\mathrm{U} 2}$, and $\operatorname{sog}^{6}$ ). A similar expansion of the dorsal rho stripe is observed in embryos with two copies of $\operatorname{sog}$ and four copies of $d p p$ (i.e., $\operatorname{sog}^{+}$; Dpdpp). (C) The dorsal rho domain in a $\operatorname{sog} /$ Y $(0 \times$ sog) embryo expands to 30-34 cells across. The genotype of this embryo can be scored as sog $/ \mathrm{Y}$ as it is not labeled by the biotin-sog probe (see inset). (D) Ectopic expression of zen in the mesoderm of a heat-shocked $4 \times$ HS-dpp embryo in a wild-type sog background (i.e., $2 \times$ sog). zen expression in the mesoderm is confined to a narrow stripe (zero to three cells wide) running along the ventral midline and to two wedge-shaped patches at the anterior and posterior ends of the mesoderm. $|E|$ Ectopic zen expression in the mesoderm of a heat-shocked sog ${ }^{-} /+$; $4 \times$ HS-dpp embryo (i.e., $1 \times \operatorname{sog})$ is significantly broader $\left(10-12\right.$ cells wide) than in similarly treated $\operatorname{sog}^{+} / \operatorname{sog}^{+} ; 4 \times \mathrm{HS}^{-d p p} \mathrm{embryos}$ $(D)$. This embryo derives from a cross of $8 \times \mathrm{HS}$ - $d p p$ males to $s o g^{\mathrm{U} 2} / \mathrm{FM} 7 \mathrm{c}$ females. The genotype of this embryo can be determined by virtue of the fact that there is a much more severe ectopic zen phenotype in the neuroectoderm of $\operatorname{sog}{ }^{\prime} / \mathrm{Y} ; 4 \times \mathrm{HS}-d p p$ embryos (see $F$ and also Fig. $3 F$ ) and because there is no such intermediate class of embryos observed in a parallel cross of $8 \times$ HS- $d p p$ males to wild-type females $(D) .(F)$ zen expression occupies the entire trunk mesoderm (18 cells wide) and neuroectoderm of a heat-shocked $\operatorname{sog}^{-} / \mathrm{Y} ; 4 \times$ HS- $d p p$ embryo (i.e., $0 \times \operatorname{sog}$ ). Ectopic zen expression is never observed in the terminal regions of the embryo, although pole cells are frequently labeled. The genotype of these embryos was determined by parallel double label experiments using a combination of zen and $\operatorname{sog}$ probes.

bryos carrying four copies of $d p p(\mathrm{Dp} d p p)$. In $\operatorname{sog}^{-} / \mathrm{Y}$ $(0 \times \operatorname{sog})$ mutants, the dorsal rho stripe expands to a domain 30-34 cells across (Fig. 5C; François et al. 1994).

The above data are consistent with Sog diffusing dorsally 12-to 15-cell diameters from the lateral source of sog RNA to determine the limit of dorsal rho expression. In addition, we observed a dose-dependent effect of sog on zen expression in the ventral region of HS- $d p p$ blastoderm embryos, suggesting that Sog also can diffuse ventrally. Thus, in wild-type embryos (i.e., $2 \times \operatorname{sog}$ ) carrying either four or eight copies of the HS- $d p p$ construct, ectopic zen expression is restricted to two wedge-shaped patches near the anterior and posterior ends of the trunk mesoderm (Fig. 5D). In more extreme examples, these patches can be joined by a narrow ventral midline stripe one to three cells wide. In $\operatorname{sog}^{-} /+$; HS-dpp embryos (i.e.,
$1 \times \operatorname{sog}$ ), the ventral zen domain expands to $\sim 12$ cells across (Fig. 5E), and in $\operatorname{sog}^{-} /$Y embryos (i.e., $0 \times \operatorname{sog}$ ), the full extent of the trunk mesoderm (18 cells) expresses zen (Fig. 5F). These data are consistent with Sog diffusing from the neuroectoderm into the presumptive mesoderm to interfere with Dpp signaling. It appears that Sog effectively inhibits Dpp signaling up to the ventral midline in wild-type embryos (approximately nine cells away from the sog expression domain), but is only able to do so over a distance of approximately three cells when the dose of $s o g$ is reduced twofold. Thus, Sog may diffuse both dorsally and ventrally from the lateral region, where it is produced, to influence the extent of gene expression domains. In addition, as the effect of $s o g$ is clearly dose dependent, it is likely that there is a gradient of Sog activity in both dorsal and ventral regions of the 
embryo. Because Sog seems to function primarily or exclusively by antagonizing Dpp signaling (see above), it may create a reciprocal gradient of Dpp activity in the dorsal region of the embryo. In this respect, Sog displays many features of a classic morphogen.

\section{Discussion}

\section{dpp promotes dorsal gene expression and suppresses} neural gene expression dorsally

Previous experiments (Irish and Gelbart 1987; Ray et al. 1991; François et al. 1994) and data presented in this paper reveal two functions of Dpp signaling in the dorsal region of the blastoderm embryo. The first is a positive action of Dpp to maintain expression of dorsally expressed genes such as zen (Ray et al. 1991), and at peak levels, to trigger expression of amnioserosal markers such as the dorsal rho stripe (François et al. 1994; Fig. $4 \mathrm{C}, \mathrm{D})$. The second role of Dpp signaling is to suppress expression of neuroectodermal genes such as l'sc (Fig. 4G). This latter role of Dpp activity dramatically influences the extent of neuroblast formation during gastrulation (Fig. 2; Wharton et al. 1996). Although $d p p$ mutants generate large numbers of ectopic neuroblasts dorsally, the final result is a hypertrophied rather than an enlarged nervous system. The basis for the ultimate nervous system defect remains to be determined.

\section{sog prevents Dpp from autoactivating} in the neuroectoderm

Our results reveal a very early role of $s o g$ in opposing $d p p$ signaling within the neuroectoderm well before gastrulation begins. sog opposes both the autoactivating and neural suppressive functions of Dpp in the neuroectoderm (Fig. 6). In $\operatorname{sog}^{-}$; Dpdpp embryos, Dpp, which presumably diffuses laterally from the dorsal region, autoactivates $d p p$ expression in the neuroectoderm. Such unopposed Dpp activity in the neuroectoderm induces expression of the dorsal markers zen and tld. Similarly, ectopic $d p p$ produced in $s o g^{-}$; HS- $d p p$ embryos induces zen expression throughout the neuroectoderm and causes the dorsal rho stripe to expand partway into the neuroectoderm. The fact that the dorsal rho stripe extends only into the dorsal portion of the neuroectoderm in $s o g^{-}{ }^{-}$, Dpdpp or $s o g^{-}$; HS-dpp embryos indicates that these embryos retain sufficient $\mathrm{D}-\mathrm{V}$ polarity to subdivide the enlarged dorsal ectoderm into dorsal-most $(r h o+z e n)$ and ventral (zen only) domains.

sog also prevents Dpp signaling from suppressing neurogenesis in lateral cells. In $\operatorname{sog}^{-}$; Dpdpp embryos, expression of l'sc is confined to a narrow ventral strip of neuroectoderm. These embryos give rise to only the inner row of scrt-expressing neuroblasts. These inner-row neuroblasts appear to be specified by a Dpp-insensitive mechanism as they are largely immune to even the most extreme conditions of ectopic Dpp expression (i.e., $\operatorname{sog}^{-}$; HS- $d p p$ embryos).

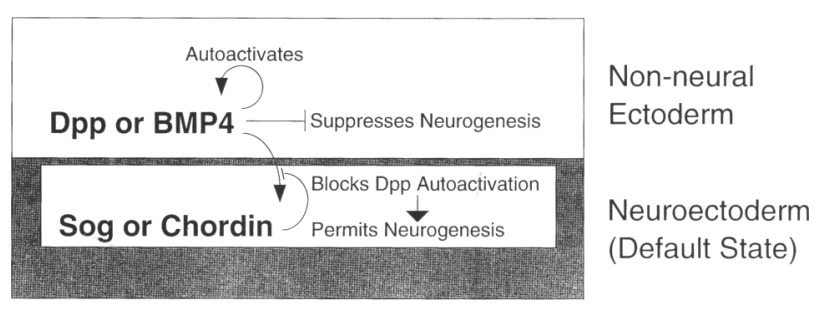

Figure 6. A model for how Sog and Chordin block Dpp and BMP-4 from autoactivating and suppressing neurogenesis in the neuroectoderm. It is inherently difficult to limit expression of genes such as $d p p$ or BMP-4 that encode diffusible autoactivating molecules. We propose that the key phylogenetically conserved function of Sog and Chordin is to protect the neuroectoderm from the invasive positive feedback loop resulting from Dpp/BMP-4 diffusion and autoactivation. The maternal mechanisms by which $s o g$ and $d p p$ are activated in abutting territories in fly embryos ii.e., direct transcriptional threshold responses to the Dorsal morphogen gradient) appear to differ fundamentally from those leading to the complementary activation of chordin and BMP-4 in frog embryos (i.e., Wnt and Activinlike induction of goosecoid expression in the Spemann organizer leading to chordin expression dorsally, and FGF plus a low Activin-like signal inducing BMP-4 expression ventrally). In contrast, once the primary zygotic response genes sog/chordin and $d p p / B M P-4$ are expressed in abutting territories, they specify neural vs. non-neural ectoderm through a highly conserved mechanism. The Dpp/BMP-4 signaling pathway plays two major roles in establishing non-neural cell fates: (1) potent suppression of neurogenesis and (2) autoactivation to maintain the expression of $d p p(B M P-4)$ and associated genes (e.g., zen and tld in flies|. Sog or Chordin prevent Dpp or BMP-4, which diffuse into the neuroectoderm from neighboring cells, from reaching the threshold level necessary to trigger autoactivation. Because neural suppression has a lower threshold for Dpp signaling than does dorsalization, Dpp/BMP-4 signaling will suppress neurogenesis once the threshold required for autoactivation is achieved (e.g., as occurs in $\operatorname{sog}^{-}$; Dpdpp fly embryos).

The hypothesis that Sog functions in the neuroectoderm to block a chain of Dpp diffusion followed by autoactivation provides a potential explanation for the high degree of evolutionary conservation between $s o g$ and $d p p$ and their vertebrate counterparts (see below). Dpp diffusion coupled to autoactivation constitutes an intrinsically invasive positive feedback loop. If unopposed, this action of Dpp would force all ectodermal cells to express $d p p$, even though $d p p$ expression is initiated in a localized pattern (Fig. 6).

\section{Distinct thresholds of Dpp signaling trigger neural suppression versus dorsalization}

We have observed consistently that significantly less $d p p$ activity is required to suppress expression of neural markers such as I'sc, scrt, and sna than to activate or maintain expression of dorsal target genes such as zen or tld. This relation appears to hold in both the lateral and dorsal regions of the embryo. For example, potent neural 
suppression is observed in the neuroectoderm under mild conditions that do not induce ectopic expression of zen. Similarly, in the dorsal region of the blastoderm embryo, we have identified a threshold of Dpp signaling (e.g., in $\mathrm{scw}^{-}$or HS-sog embryos) that is largely adequate for suppressing l'sc expression dorsally but is insufficient for maintaining zen expression.

\section{Dpp and Sog consolidate pattern initiated by the Dorsal morphogen gradient}

Because the protective action of $s o g$ in the neuroectoderm is only strictly required in embryos having elevated levels of Dpp, parallel pathways are likely to act together with sog to block Dpp from autoactivating in the neuroectoderm. The maternal morphogen Dorsal is one likely candidate for such a parallel pathway as ectopic $d p p$ expression is observed in the neuroectoderm of $\operatorname{sog}^{-}$(Fig. $3 \mathrm{H})$ or Dpdpp embryos derived from $\mathrm{dl}^{-} /+$mothers. Dorsal, an NF-kB-related transcription factor, activates expression of key patterning genes in ventral and lateral regions of the embryo but represses expression of several ZDG genes including $d p p$ in these same cells (Huang et al. 1993, 1995; Jiang et al. 1993|. Data presented here suggest that sog stabilizes the primary subdivision of the ectoderm into neural versus non-neural components by Dorsal. Dpp activity in dorsal cells exerts a similar stabilizing influence on primary maternal patterning as Dpp maintains expression of several ZDG genes while suppressing expression of genes promoting the alternative neuroectodermal fate.

Another example of parallel processing is that $d p p$ is not required dorsally to maintain expression of ZDG genes such as tld or $d p p$ itself, although ectopic expression of $d p p$ in the neuroectoderm is sufficient for inducing expression of these genes. Similarly, although $d p p$ function is not required to set the limits of the lateral rho stripe, ectopic $d p p$ expression reduces the width of this domain. This suggests that Dpp diffusing laterally from the dorsal domain works in parallel with Dorsal (Ip et al. 1992) to determine the dorsal limit of the lateral rho stripe in the neuroectoderm. Thus, primary Dorsal response genes, such as $\operatorname{sog}$ and $d p p$, function in part to stabilize and maintain the primary D-V subdivisions defined initially by Dorsal.

\section{Sog may be a dedicated Dpp antagonist}

The observation that the $\operatorname{sog}^{-} ; d p p^{-}$double mutant phenotype is indistinguishable from that of $d p p^{-}$single mutants is consistent with sog functioning as a dedicated antagonist of Dpp signaling. The absence of any aggravating effect of ectopic sog expression on the $d p p^{-}$ phenotype also supports models in which sog functions specifically to block Dpp action. Although it is possible that there are markers that would reveal a stronger phenotype in HS-sog; $d p p^{-}$embryos than in $d p p^{-}$single mutants, l'sc, tld, and zen should be sensitive indicators of such a difference as they are either expressed normally or misexpressed to only a limited extent in $d p p^{-}$single mutants.

Recently it has been shown that Chordin, the vertebrate homolog of Sog, binds to BMP-4 (the vertebrate homolog of Dpp) (Picolla et al. 1996). If Sog functioned by a similar mechanism, one might expect that ectopic expression of $\operatorname{sog}$ could phenocopy $\mathrm{dpp}^{-}$mutants. The most extreme phenotype resulting from ubiquitous sog expression we observed, however, is substantially weaker (i.e., comparable to that of $s c w^{-}$mutants). Similarly, analysis of cuticle patterns suggested that dorsal injection of maximal quantities of $\operatorname{sog}$ mRNA generates ventralized phenotypes significantly less extreme than observed in $d p p^{-}$embryos (Holley et al. 1995). These data do not support simple models in which Sog binds and sequesters all forms of Dpp in inactive complexes. Although it is possible that the amounts of ectopic sog produced by $8 \times$ HS-sog and by $\operatorname{sog}$ mRNA injection are insufficient to bind all available Dpp, we note that maximal $8 \times$ HS-sog phenotypes can also be obtained at similar frequencies and with similar penetrance in $4 \times$ HS$\operatorname{sog}$ embryos, and that increasing the amount of sog mRNA injected into embryos does not increase the severity of the cuticular phenotype (Holley et al. 1995). This is in contrast to the extreme dosage-sensitive interactions observed between $d p p$ and sog mutants (Ferguson and Anderson 1992b; Wharton et al. 1993; François et al. 1994; Yu et al. 1996). Tentatively, we favor models in which an active form of Dpp is insensitive to inhibition by Sog, or in which Sog interferes with the action of molecules potentiating Dpp activity or propagating the Dpp signal. Consistent with this view, we have obtained recent evidence that $\operatorname{sog}$ may block only one of two effects of Dpp signaling during wing vein development (Yu et al. 1996).

\section{Sog has properties of a morphogen}

In this paper we extended previous observations suggesting that Sog not only functions locally to block Dpp signaling in the neuroectoderm, but that it also diffuses long distances to influence gene expression in neighboring cells (Zusman et al. 1988; François et al. 1994). We observed that the width of the dorsal rho stripe depends on the dose of sog, and similarly, that the extent of the ventral stripe of ectopic zen expression in HS- $d p p$ embryos is sensitive to sog dosage. These data are compatible with Sog diffusing 12- to 15-cell diameters dorsally to establish a gradient of Dpp activity. In this sense Sog could be considered a morphogen. It is also possible, however, that other genes function in parallel with sog to determine the limits of target gene expression. If this were true, one could imagine that $\operatorname{sog}$ functions only as an on-off switch to reveal existing prepatterns in responding dorsal or ventral cells. Although we have no direct evidence to contradict this possibility, there are two reasons why it seems unlikely. First, the dorsal rho 
stripe and the ventral zen stripe (in HS- $d p p$ embryos) assume three different diameters in embryos carrying two, one, or zero doses of sog. In the simple case that sog were functioning as a binary on-off switch to trigger gene expression according to a prepattern of sensitive cells (e.g., dorsal ectoderm) versus insensitive cells (e.g., amnioserosa), one would expect two, not three, potential boundaries. Second, there is no known basis for suspecting the existence of prepatterns in either the dorsal or ventral regions of the blastoderm embryo. In fact, current data suggest that all cells in the dorsal region are equivalent and specified as such by the absence of nuclear Dorsal. Direct visualization of the pattern of Sog proteins will help resolve this question.

In summary, Sog and Dpp act locally to consolidate the primary subdivision of the embryo into neural versus non-neural ectoderm. These genes also function nonautonomously to trigger the next round of subdivision within each of these domains.

\section{Mechanisms for subdividing the ectoderm into neural versus nonneural components have been conserved during evolution}

From an evolutionary point of view, it is striking that the Xenopus BMP-4 and chordin genes, like $d p p$ and sog, are expressed in abutting D-V domains (François et al. 1994; Graff et al. 1994; Maeno et al. 1994; Sasai et al. 1994; Schmidt et al. 1995b) and encode molecules highly related to $d p p$ and sog (François and Bier 1995), respectively. These vertebrate and invertebrate genes play homologous roles during Xenopus and Drosophila embryogenesis (Padgett et al. 1993; Holley et al. 1995; Schmidt et al. 1995a). There are also strong parallels between the biological actions of these homologous gene sets. Thus, BMP-4 has been shown to function as a potent neural suppressor during Xenopus development (Sasai et al. 1995; Schmidt et al. 1995b; Wilson and Hemmati-Brivanlou 1995) and is capable of autoactivating in Xenopus (Jones et al. 1992; Schmidt et al. 1996). The data presented here reinforce the view that mechanisms for subdividing the ectoderm into neural versus non-neural territories have been conserved in detail during the course of evolution (Padgett et al. 1993; Wilson and HemmatiBrivanlou 1995; François and Bier 1995; Holley et al. 1995; Sasai et al. 1995; Schmidt et al. 1995a; Fig. 6).

\section{Materials and methods}

Fly stocks

The $\operatorname{sog}^{\mathrm{U} 2}$ and $\operatorname{sog}^{\mathrm{P} 1}$ alleles are described in François et al. (1994). The $\operatorname{sog}^{6}$ allele, (Wieschaus et al. 1984) was obtained from the Bloomington Indiana Stock Center. $\operatorname{sog}^{\mathrm{U} 2}$ and $\operatorname{sog}^{6}$ are RNA null alleles (François et al. 1994). The CyODpdpp chromosome (CyO23, kindly supplied by W. Gelbart, Harvard University/ carries a genomic $d p p$ transgene expressed as the wildtype gene during early embryogenesis in addition to a wild-type copy of the $d p p$ locus. The $8 \times$ HS- $d p p$ stock was kindly provided by Dr. Ronald K. Blackman (University of Illinois, Urbana). Construction of the $8 \times$ HS-sog line is described in Yu et al. (1996). Other balancers and chromosomal markers (Lindsley and Grell 1968; Lindsley and Zimm 1992/ were obtained from either the Bloomington Indiana Stock Center or the Bowling Green Stock Center.

Scoring the genotype of $\operatorname{sog}^{-}$; Dpdpp and $\operatorname{sog}^{-}$; $\mathrm{dpp}^{-}$embryos

To identify embryos of the genotype $\operatorname{sog}^{-} / \mathrm{Y} ; \mathrm{Dp} d p p / \mathrm{Dp} d p p$ in collections from $s \mathrm{~g}^{\mathrm{U} 2} / \mathrm{FM} 7 \mathrm{c}_{i}$ Dfdpp/CyODpdpp parents, embryos were either triple hybridized with probes for rho (digoxigenin labeled), $d p p$ (digoxigenin labeled), and sog (biotin labeled), or were double stained for l'sc or scrt (digoxigenin labeled) and $s o g$ (biotin labeled) and scored for $d p p$ function by the severe gastrulation phenotype typical of $d p p^{-}$mutants. These experiments revealed premature ectopic dorsal expression of rho in $\operatorname{sog}^{-}$; Dpdpp embryos (i.e., such embryos did not label with a $s o g$ probe but did label with a $d p p$ probe). Similar experiments without addition of the $d p p$ probe were also performed on stocks carrying a null $d p p$ point mutant allele $\left(\operatorname{sog}^{\mathrm{U} 2} / \mathrm{FM} 7 \mathrm{c}\right.$; $d p p^{\text {hin }} /$ CyODp $d p p$ ). The $s o g^{-}$; Dpdpp embryos shown in Figure 2 were obtained from parallel single label experiments with lower background signals as initial experiments revealed that $s o g^{-}$; Dpdpp embryos could be identified unambiguously by expression of the marker genes alone. In the multilabel experiments described above, it was also possible to identify unambiguously $\operatorname{sog}^{-} ; d p p^{-}$double mutant embryos as shown in Figure 4 .

Double label in situ hybridization

Double in situ hybridization with digoxigenin- and biotin-labeled RNA probes is described in detail in $\mathrm{O}^{\prime} \mathrm{Neill}$ and Bier (1994).

\section{Acknowledgments}

We thank Ronald K. Blackman and Richard Padgett for providing the $8 \times$ HS- $d p p$ stock and Kweon Yu for the $8 \times$ HS-sog and $4 \times$ HS-sog; $d p p^{-/ C y o D p d p p ~ s t o c k s . ~ W e ~ t h a n k ~ M i c h a e l ~ L e v i n e, ~}$ Karen Lunde, Bill McGinnis, Peter Gergen, David Kimelman, and Kweon Yu for critical comments on the manuscript, and Kathryn S. Burton for assembling the figures. This work was supported by National Institutes of Health Grant No. RO1NS29870, and Research Grant No. 5-FY92-1175 from the March of Dimes Birth Defects Foundation. V.F. was supported in part by grants from the Centre National de la Recherche Scientifique and the North Atlantic Treaty Organization. E.B. was supported by funds from the McKnight Neuroscience Foundation, Sloan Foundation, and an American Cancer Society Junior Faculty Award.

The publication costs of this article were defrayed in part by payment of page charges. This article must therefore be hereby marked "advertisement" in accordance with 18 USC section 1734 solely to indicate this fact.

\section{References}

Arora, K. and C. Nüsslein-Volhard. 1992. Altered mitotic domains reveal fate map changes in Drosophila embryos mutant for zygotic dorsoventral patterning genes. Development 114: 1003-1024.

Bier, E., L.Y. Jan, and Y.N. Jan. 1990. rhomboid, a gene required for dorsoventral axis establishment and peripheral nervous system development in Drosophila melanogaster. Genes \& 
Dev. 4: 190-203.

Ferguson, E.L. and K.V. Anderson. 1992a. Decapentaplegic acts as a morphogen to organize dorsal-ventral pattern in the Drosophila embryo. Cell 71: 451-461.

- 1992b. Localized enhancement and repression of the activity of the TGF- $\beta$ family member, decapentaplegic, is necessary for dorsal-ventral pattern formation in the Drosophila embryo. Development 114: 583-597.

François, V. and E. Bier. 1995. The Xenopus chordin and Drosophila short gastrulation genes encode homologous proteins functioning in dorsal-ventral axis formation. Cell 80: 19-20.

François, V., M. Solloway, J.W. O'Neill, J. Emery, and E. Bier. 1994. Dorsal-ventral patterning of the Drosophila embryo depends on a putative negative grow th factor encoded by the short gastrulation gene. Genes \& Dev. 8: 2602-2616.

Graff, J.M., R.S. Theis, J.J. Song, A.J. Celeste, and D.A. Melton. 1994. Studies with a BMP receptor suggest that ventral mesoderm-inducing signals override dorsal signals in vivo. Cell 79: 169-179.

Holley, S.A., P.D. Jackson, Y. Sasai, B. Lu, E.M. De Robertis, F.M. Hoffmann, and E.L. Ferguson. 1995. A conserved system for dorsal-ventral patterning in insects and vertebrates involving sog and chordin. Nature 376: 249-253.

Huang, J.D., D.H. Schwyter, J.M. Shirokawa, and A.J. Courey. 1993. The interplay between multiple enhancer and silencer elements defines the pattern of decapentaplegic expression. Genes \& Dev. 7: 694-704.

Huang, J.D., T. Dubnicoff, G.J. Liaw, Y. Bai, S.A. Valentine, J.M. Shirokawa, J.A. Lengyel, and A.J. Courey. 1995. Binding sites for transcription factor NTF-1/Elf-1 contribute to the ventral repression of decapentaplegic. Genes \& Dev. 9: 3177 3189.

Ip, Y.T., R.E. Park, D. Kosman, E. Bier, and M. Levine. 1992. The dorsal gradient morphogen regulates stripes of rhomboid expression in the presumptive neuroectoderm of the Drosophila embryo. Genes \& Dev. 6: 1728-1739.

Irish, V.F. and W.M. Gelbart. 1987. The decapentaplegic gene is required for dorsal-ventral patterning of the Drosophila embryo. Genes \& Dev. 1: 868-879.

Jiang, J., H. Cai, Q. Zhou, and M. Levine. 1993. Conversion of a dorsal-dependent silencer into an enhancer: Evidence for dorsal corepressors. EMBO I. 12: 3201-3209.

Jones, C.M., K.M. Lyons, P.M. Lapan, C.V.E. Wright, and B.L.M. Hogan. 1992. DVR-4 (bone morphogenetic protein-4) as a posterior ventralizing factor in Xenopus mesoderm induction. Development 115: 639-647.

Kingsley, D.M. 1994. The TGF-beta superfamily: new members, new receptors, and new genetic tests of function in different organisms. Genes \& Dev. 8: 133-146.

Kingsley, D.M., A.E. Bland, J.M. Grubber, P.C. Marker, L.B. Russell, N.G. Copeland, and N.A Jenkins. 1992. The mouse short ear skeletal morphogenesis locus is associated with defects in a bone morphogenetic member of the TGF beta superfamily. Cell 71: 399-410.

Lindsley, D.L. and E.H. Grell. 1968. Genetic variations in Drosophila melanogaster. Carnegie Institute of Washington, Washington, DC.

Lindsley, D.L. and G.G. Zimm. 1992. The genome of Drosophila melanogaster. Academic Press, San Diego, CA.

Maeno, M., R.C. Ong, A. Suzuki, N. Ueno, and H.K. Kung. 1994. A truncated bone morphogenetic protein receptor alters the fate of ventral mesoderm to dorsal mesoderm-Roles of animal tissue in the development of ventral mesoderm. Proc. Natl. Acad. Sci. 91: 10260-10264.

O'Neill, J.W. and E. Bier. 1994. Double in situ hybridization using biotin and digoxigenin tagged RNA probes. BioTech- niques 17: 870-875.

Padgett, R.W., R.D. St. Johnson, and W.M. Gelbart. 1987. A transcript from a Drosophila pattern gene predicts a protein homologous to the transforming growth factor- $\beta$ family. $\mathrm{Na}$ ture 325: 81-84.

Padgett, R.W., I.M. Wozney, and W.M. Gelbart. 1993. Human BMP sequences can confer normal dorsal-ventral patterning in the Drosophila embryo. Proc. Natl. Acad. Sci. 90: 29052909.

Picolla, S., Y Sasai, B. Lu, and E.M. de Robertis. 1996. Dorsoventral patterning in Xenopus: Inhibition of ventral signals by direct binding of chordin to BMP-4. Cell 86: 589-598.

Ray, R.P., K. Arora, C. Nüsslein-Volhard, and W.M. Gelbart. 1991. The control of cell fate along the dorsal-ventral axis of the Drosophila embryo. Development 113: 35-54.

Rosen, V. and R.S. Thies. 1992. The BMP proteins in bone formation and repair. Trends Genet. 8: 97-102.

Roth, S., D. Stein, and C. Nüsslein-Volhard. 1989. A gradient of nuclear localization of the Dorsal protein determines dorsoventral pattern in the Drosophila embryo. Cell 59: 11891202.

Rushlow, C.A., K. Han, J.L. Manley, and M. Levine. 1989. The graded distribution of the dorsal morphogen is initiated by selective nuclear transport in Drosophila. Cell 59: 11651177.

Sasai. Y., B. Lu, H. Steinbeisser, D. Geissert, L. Gont, and E.M. De Robertis. 1994. Xenopus chordin: A novel dorsalizing factor activated by organizer specific homeobox genes. Cell 79: 779-790.

Sasai, Y., B. Lu, H. Steinbeisser, and E.M. De Robertis. 1995. Regulation of neural induction by the Chd and BMP-4 antagonistic patterning signals in Xenopus. Nature 376: 333336.

Schmidt, J., V. François, E. Bier, and D. Kimelman. 1995a. The Drosophila short gastrulation gene induces an ectopic axis in Xenopus: Evidence for conserved mechanisms of dorsalventral patterning. Development 121: 4319-4328.

Schmidt, J., A. Suzuki, N. Ueno, and D. Kimelman. 1995b. Localized BMP-4 mediates dorsal-ventral patterning in early the Xenopus embryo. Dev. Biol. 169: 37-50.

Schmidt, J.E., G. Vondassow, and D. Kimelman. 1996. Regulation of dorsal-ventral patterning- the ventralizing effects of the novel Xenopus homeobox gene vox. Development 122: $1711-1721$.

St. Johnson, R.D. and W.M. Gelbart. 1987. Decapentaplegic transcripts are localized along the dorsal-ventral axis of the Drosophila embryo. EMBO I. 6: 2785-2791.

Steward, R. 1989. Relocalization of the dorsal protein from the cytoplasm to the nucleus correlates with its function. Cell 59: 1179-1188.

Storm, E.E., T.V. Huynh, N.G. Copeland, N.A. Jenkins, D.M. Kingsley, and S.J. Lee. 1994. Limb alterations in brachypodism mice due to mutations in a new member of the TGF beta-superfamily. Nature 368: 639-643.

Tatei, K., H. Cai, Y.T. Ip, and M. Levine. 1995. Race: A Drosophila homologue of the angiotensisn converting enzyme. Mech. Dev. 51: 157-168.

Wieschaus, E., C. Nüsslein-Volhard, and G. Jürgens. 1984. Mutations affecting the pattern of the larval cuticle in Drosophila melanogaster. II. Zygotic loci on the X-chromosome and fourth chromosome. Roux's Arch. Dev. Biol. 193: 296307.

Wilson, P.A. and A. Hemmati-Brivanlou, A. 1995. Induction of epidermis and inhibition of neural fate by BMP-4. Nature 376: 331-333.

Wharton, K.A., R.P. Ray, and W.M. Gelbart. 1993. An activity 
Biehs et al.

gradient of decapentaplegic is necessary for the specification of dorsal pattern elements in the Drosophila embryo. Development 117: 807-822.

Wharton, K.A., R.P. Ray, S.D. Findley, H.E. Duncan, and W.M. Gelbart. 1996. Molecular lesions associated with alleles of decapentaplegic identify residues necessary for TGF- $\beta$ /BMP signaling in Drosophila melanogaster. Genetics 142: 493505.

Yu, K., M.A. Sturtevant, B. Biehs, V. François, R.W. Padgett, R.K. Blackman, and E. Bier. 1996. The Drosophila decapentaplegic and short gastrulation genes function antagonistically during wing vein development. Development 122: 4033-4044.

Zusman, S.B., D. Sweeton, and E.F. Wieschaus. 1988. short gastrulation, a mutation causing delays in stage specific cell shape changes during gastrulation in Drosophila melanogaster. Dev. Biol. 129: 417-427. 


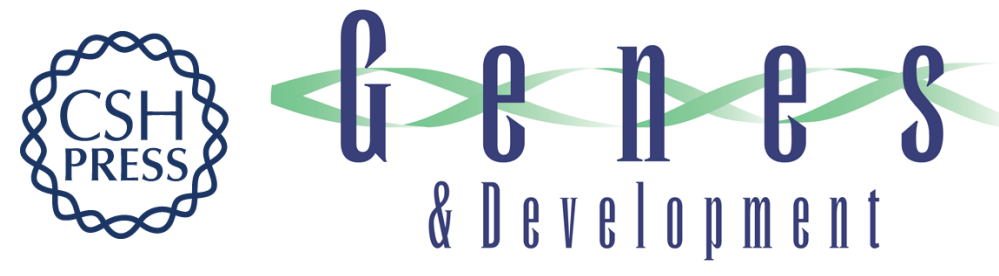

\section{The Drosophila short gastrulation gene prevents Dpp from autoactivating and suppressing neurogenesis in the neuroectoderm.}

B Biehs, V François and E Bier

Genes Dev. 1996, 10:

Access the most recent version at doi:10.1101/gad.10.22.2922

References This article cites 40 articles, 18 of which can be accessed free at:

http://genesdev.cshlp.org/content/10/22/2922.full.html\#ref-list-1

License

Email Alerting

Service

Receive free email alerts when new articles cite this article - sign up in the box at the top right corner of the article or click here.

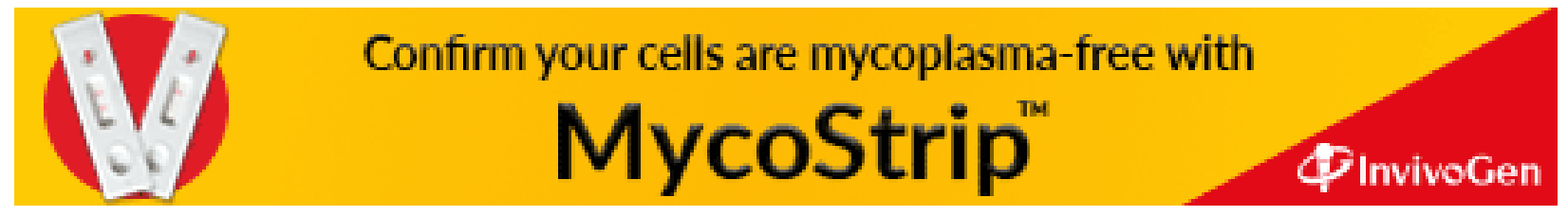

